\title{
IMPACT OF INSTANTANEOUS CONTROLLED PRESSURE PROP (DIC) TECHNOLOGY ON EXTRACTION OF TOTAL PHENOLS OF MOROCCAN SALVIA OFFICINALIS
}

\author{
MHARTI Fatima-Zohra ${ }^{a, b}$, ABDELLAOUI Abdelfattah ${ }^{a}$, KAMAL Ibtisam $^{c}$, LYOUSSI Badiaa $^{a}$, \\ ALLAF Tamara ${ }^{\mathrm{d}}$, and ALLAF Karim ${ }^{\mathrm{b}}$
}

a: University Sidi Mohamed Ben Abdellah, Faculty of Science, Department of Biology, Laboratory of

Physiology, Pharmacology and Environmental Health. B.P. 1796 Atlas, Fez. Morocco

mhartifaty@gmail.com

b: University of La Rochelle, Intensification of Transfer Phenomena on Industrial Eco-Processes, Laboratory

Engineering Science for Environment LaSIE FRE 3474 CNRS, 17042 La Rochelle, France

kallaf@univ-Ir.fr

c: SoranUniversity, Faculty of Science, Soran-Erbil, Kurdistan Region, Iraq.

ibtisamkamal@yahoo.fr

d: ABCAR DIC Process, B.P. 1205317010 cedex 01 La Rochelle, France.

tamara.allaf@abcar-dic.com

\begin{abstract}
The main objective of this study was to intensify the extraction of total phenols from Salvia officinalis $L$. using instant controlled pressure drop (DIC) as a texturing pre-treatment. The effect of solvent type on Total Phenolic Content (TPC) was also studied. TPC was determined using spectrophotometric Folin-Ciocalteau method and external calibration with Gallic acid. The obtained results showed that water was the most efficient solvent to extract total phenols from Salvia officinalis L. Moreover, texturing and expansion by DIC pretreatment had a great impact on polyphenol yields and revealed greater extraction kinetics. Likewise, drying kinetics of DIC-treated sage was enhanced compared to the raw material. DIC-assisted extraction can be considered as a promising technology to use in the case of the Moroccan Salvia officinalis L. as an important Mediterranean source of natural phenols.
\end{abstract}

\section{INDEXING TERMS/KEYWORDS}

Instant Controlled Pressure Drop (DIC), Total Phenols Content TPC, Drying Kinetics; Extraction Kinetics, Salvia officinalis L.

\section{ACADEMIC DISCIPLINE AND SUB-DISCIPLINES}

Process Engineering - Chemical Engineering; Solvent Extraction

\section{SUBJECT CLASSIFICATION}

Extraction of plant-based natural active compounds

\section{TYPE (METHOD/APPROACH)}

Research paper on Quasi-Experimental study on the intensification of process of extraction

\section{Council for Innovative Research}

Peer Review Research Publishing System

Journal: Journal of Advances in Chemistry

Vol. 7, No. 2

editor@cirworld.com

www.cirworld.com, member.cirworld.com 


\section{INTRODUCTION}

Salvia officinalis L. is an important medicinal plant widely used in popular Moroccan medicines. This Mediterranean origin plant is well known for its antioxidant properties, mainly due to its phenolic compounds[1]. It is a rich source of many useful secondary metabolite including phenolics and their derivatives that are important within various pharmacopoeias [2]. Sage extracts present strong antioxidant activity capable of increasing the stability of various foodstuffs such as vegetal oils [37]. It has been used extensively for the treatment of coronary heart disease, cerebrovascular disease, hepatitis, hepatocirrhosis, chronic renal failure, dysmenorrhea and neuroasthenic insomnia [8].

The extraction of polyphenols is the critical step in the isolation of active compoundsfrom the plant material. Conventional extraction methods are usually based on selecting the proper solvent and defining the adequate temperature in order to increase solubility of targeted compounds. The polarity of the solvent plays an important role in the extraction of phenolic compounds [9]. Polyphenols range from polar to non-polar molecules, thus a wide range of solvents has been used for their extraction such as water, ethanol/water and acetone/water mixtures [10,11]. The extraction methods often require long processing time and great solvent quantity; they often generate thermal degradation of biologically active compounds.

Instant controlled pressure drop technology (DIC) is a well-known operation, which was defined in 1988 [12], to be studied, developed, optimized and used at industrial scales for various applications. Indeed it has been over the years studied and developed as a pre-treatment for extraction of non-volatile molecules [13], as a direct extraction of volatile compounds [1416], drying, texturing [17]. DIC is a thermo-mechanical process generated by subjecting the raw material for a short period of time to high-saturated steam pressure followed by an abrupt pressure drop towards a vacuum.

In recent years much attention has been directed to the biologically active water-soluble components in the plant used in traditional medicine. This present work aims at studying the intensification of the extraction of total phenols from Moroccan sage leaves using DIC assisted extraction and optimizing its processing conditions in terms of extraction time and drying kinetics, using various solvents.

\section{MATERIALS AND METHODS \\ PLANT MATERIAL AND CHEMICALS}

Fresh leaves of Salvia officinalis were collected in July 2012 from the suburb of Fes (Morocco). Their water content was about 55\% db (Dry Basis).

All the solvents (ethanol, acetone and isobutanol) used for extraction of TPC were purchased from Carlo Erba Reagenti $\mathrm{SpA}$ (France). Gallic acid used for the determination of the antioxidant activity, was purchased from Sigma-Aldrich (Germany).

\section{TREATMENT PROTOCOL}

Just after harvesting, sage leaves were treated by DIC with different processing conditions(Figure 1).

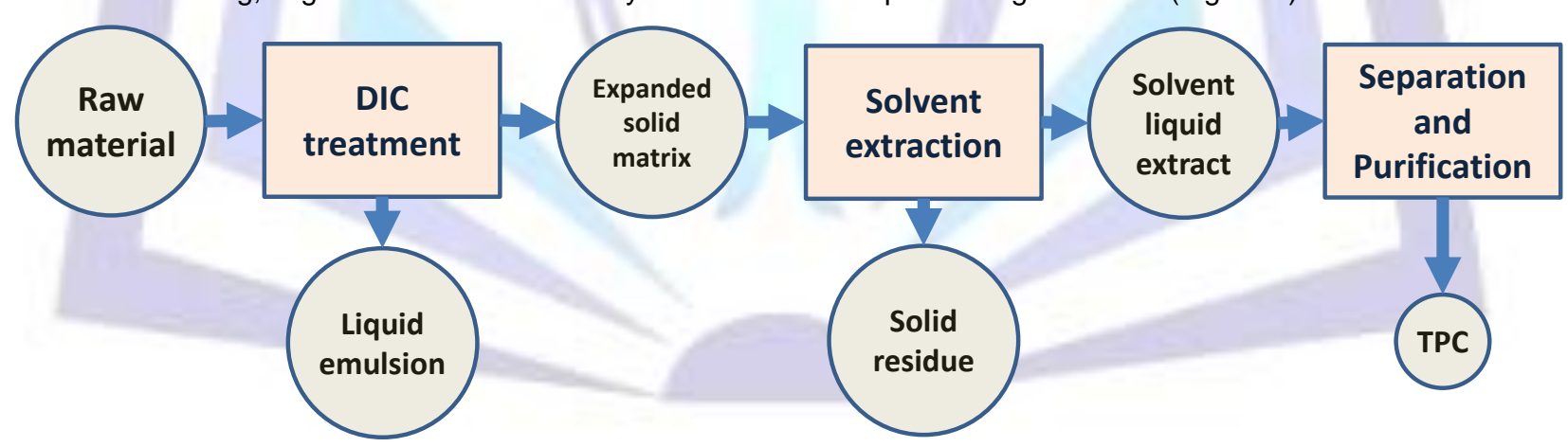

Figure 1. Schematic diagram of the TPC extraction from sage leaves.

\section{DRYING}

Sage samples (fresh and DIC treated) were dried in an oven (Firlabo: Universal Oven Air concept AC60, France), in order to achieve a thorough comparison. Drying process was carried out using an airflow dryer at $45^{\circ} \mathrm{C}$, with low relative humidity ( 1 to $2 \%$ ) high-speed (>2 m/s). The drying operation was performed to reach a water content of about $10 \% \mathrm{db}$

\section{DIC PROCESS}

In the present work, the reactor used was a new developed version of DIC apparatus (Micro-DIC provided from ABCARDIC Process, La Rochelle, France) (Figure 2) as described by [18]. 


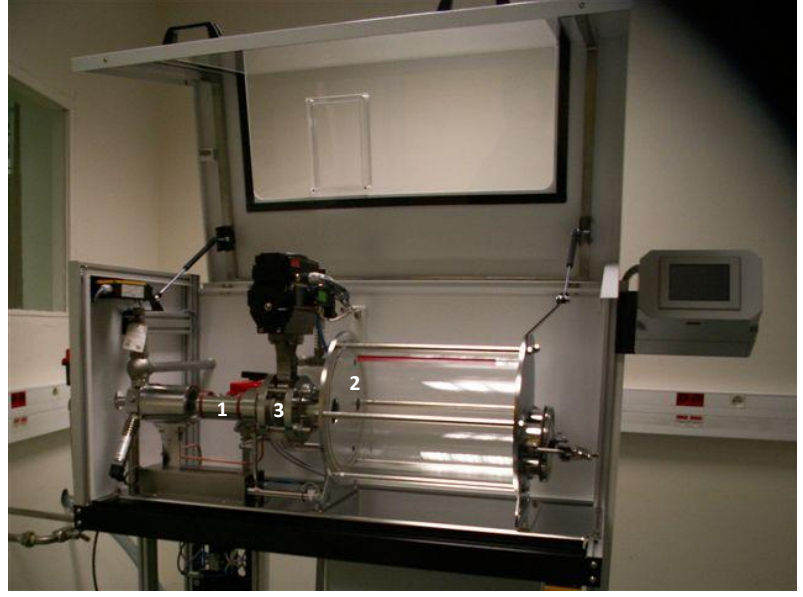

Figure 2. Schematic diagram of the DIC reactor: (1) Processing vessel; (2) transparent Jacket vacuum tank; (3) controlled instant pressure drop valve [18].

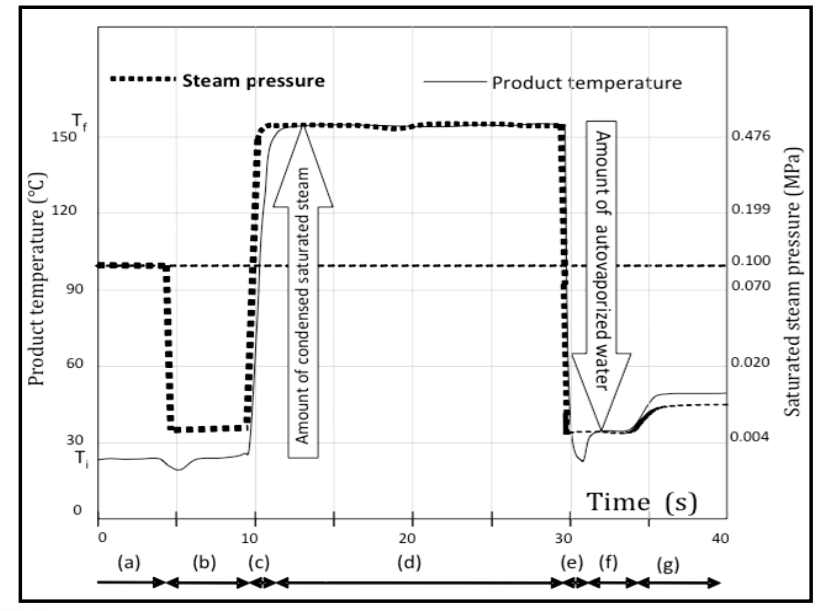

Figure 3. Different stages of a DIC treatment [18]

The treatment of plant by DIC process is conventionally composed of the three main stages (Figure 3) of:
a. Heating saturated steam to be injected in the treatment vessel;
b. High-pressure/high-temperature stage by maintaining constant the pressure P and the
temperature T for dozens of seconds (thermal treatment time t);
c. Instant pressure-drop stage to be carried out toward a vacuum.
Treatment usually starts by establishing a first vacuum stage in the DIC processing vessel where plant
sample is priory sited. This stage aims at reducing the resistance regarding the steam diffusion, thus
consequently improving the heating rate. After closing the main pneumatic valve, high-pressure steam is
injected into the reactor to keep the pressure/temperature constant during the treatment time. An abrupt
pressure drop towards a vacuum usually follows the thermal treatment stage. The resulting
autovaporization induces an instant cooling and simultaneously a possible controlled expansion of the
solid material.

\section{DESIGN OF EXPERIMENTS AND STATISTICAL PROTOCOL}

Preliminary experiments were performed with various DIC processing conditions. They aimed at defining the relevant operating parameters and their possible ranges. A specific experimental design was then defined with two DIC operating parameters (independent variables): saturated steam pressure $\mathrm{P}$ and total thermal treatment time $\mathrm{t}$, ranged between 0.2 and $0.5 \mathrm{MPa}$, and between 40 and $80 \mathrm{~s}$, respectively (Table 1). DIC treatments were achieved in order to identify the impact of operating parameters on solvent extraction of polyphenols from the expanded solid samples.

Table 1. Coded levels for independent variables used in developing experimental data.

\begin{tabular}{ccc}
\hline Coded levels & Saturated steam pressure P (MPa) & Processing time $t(s)$ \\
\hline$(-\alpha)$ & 0.2 & 40 \\
$(-1)$ & 0.24 & 46 \\
\hline central point & 0.35 & 60 \\
\hline$(+1)$ & 0.46 & 74 \\
$(+\alpha)$ & 0.5 & 80 \\
\hline value of 1 & 0.11 & 14
\end{tabular}

Statistical treatment of the data issued from the experimental work was used to study, analyze and optimize DIC treatment. 2-parameter/5-level Response surface methodology (RSM) central composite rotatable experimental design method was defined with $2^{2}=4$ factorial points; $2^{*} 2=4$ star points; and 5 replicates of central points (Table 2 ). The effects of operating parameters $\mathrm{P}$ and t on various responses (dependent variables) were studied using Statgraphics plus (1995-5.1 version, France). 
Table 2. Experimental design used in DIC treatment for TPC.

\begin{tabular}{ccc}
\hline Trial number & Saturated Steam Pressure (MPa) & Thermal Treatment Time $(\mathrm{s})$ \\
\hline DIC 1, 4, 7, 10 and 13 & 0.35 & 60 \\
DIC 2 & 0.50 & 60 \\
DIC 3 & 0.35 & 80 \\
DIC 5 & 0.46 & 74 \\
DIC 6 & 0.46 & 46 \\
DIC 8 & 0.24 & 46 \\
DIC 9 & 0.24 & 74 \\
DIC 11 & 0.20 & 60 \\
DIC 12 & 0.35 & 40 \\
Raw material & - & - \\
\hline
\end{tabular}

\section{SOLVENT EXTRACTION}

The extraction of polyphenol is influenced by several variables, such as type of solvent; the effects of solvent were investigated for determining the best condition for the extraction of polyphenol from sage.

A quantity of $0.1 \mathrm{~g}$ of raw material (used as reference) and differently DIC-treated leaves was added to $10 \mathrm{ml}$ of deionized water maintained at $25^{\circ} \mathrm{C}$. The conventional maceration was used as aqueous extraction method with stirring (400 rpm) for $40 \mathrm{~min}$. The aqueous extract was filtered immediately using $0.45 \mu \mathrm{m}$ membrane filter (Sartorius Stedim Biotech $\mathrm{GmbH} /$ Germany). The resultant filtrates were analyzed for measuring the Total Phenolic Content TPC to be used as a dependent variable (response) of the operation. The samples were analyzed in triplicate.

\section{DETERMINATION OF PHENOLIC COMPOUNDS}

Gallic acid solution was used as the standard reference to define the TPC value of extract. $0.02 \mathrm{~g}$ of Gallic acid was dissolved and diluted to $100 \mathrm{~mL}$ with absolute methanol. The solution of $200 \mu \mathrm{g} / \mathrm{mL}$ Gallic acid was obtained. Successive dilutions of Gallic acid content ranged from 10 to $200 \mu \mathrm{g} / \mathrm{mL}$ were used to develop the standard curve. The calibration equation for Gallic acid $\left(R^{2}=0.9985\right)$ is shown in equation (1):

$y=0.0083 x+0.0562($ Eq. 1$)$

TPC of each extract was estimated through Folin-Ciocalteu colorimetric method [19], with some modifications using Spectrophotometer (Helios Omega UV/VIS Thermo Scientific Merk) at $765 \mathrm{~nm} .0 .1 \mathrm{~g}$ of crude extracted was dissolved in $10 \mathrm{ml}$ of deionized water. Then test tubes with $0.5 \mathrm{~mL}$ of solution mixed with $2.0 \mathrm{~mL}$ of Folin-Ciocalteau reagent $(20 \%$, v/v) were caped and shaken for $10 \mathrm{~s}$ and put on to incubation in a water bath at $45^{\circ} \mathrm{C}$ for $5 \mathrm{~min}$. Afterwards an amount of $2 \mathrm{ml}$ of $20 \%$ of sodium carbonate solution was added into the mixture to be left at room temperature for 30 min. TPC was determined and expressed in terms of Gallic Acid Equivalent dry basis (mg GAE/g db). Each experiment was carried out in a triplicate.

\section{SCANNING ELECTRON MICROSCOPE SEM}

The sage leaf microstructure of untreated and DIC treated samples were studied using a Scanning electron microscope (SEM) environmental type JEOL model JSM5410LVFEI Quanta 200F Scanning Electron Microscope (Philips Croissy-surSeine; France). The samples were placed on a covered support using carbon adhesive; and scanned in a partial vacuum $7 \mathrm{~Pa}$ with $20 \mathrm{kV}$ acceleration tension. SEM pictures enabled to highlight the impact of DIC as a texturing treatment.

\section{RESULTS AND DISCUSSION DRYING KINETICS}

The drying kinetics time was studied for untreated and DIC treated sage leaves (Figure 4).The obtained results showed that the DIC treated samples had a quick drying kinetics compared to untreated material. As shown in figure 3 , drying time of all DIC samples were much shorter than that of raw material. The time necessary to let the water content reach $12 \%$ dry basis was $540 \mathrm{~min}$ for control while it was $15 \mathrm{~min}$ for DIC 4 at $0.46 \mathrm{MPa}$ for $74 \mathrm{~s}$. 

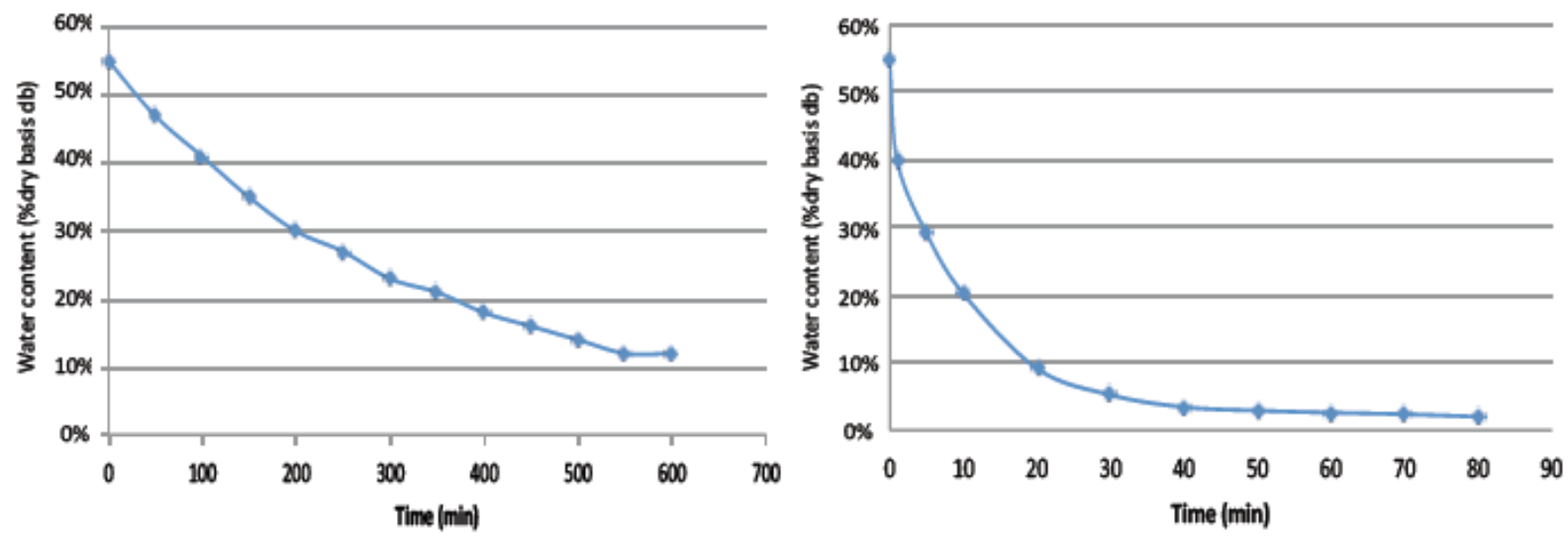

Figure 4. Drying kinetics at $45^{\circ} \mathrm{Cof}$ (left) fresh sage leaves ascontrol (Raw-Material) and (right) DIC-5 treated samples.

\section{EXTRACTION PROCESS}

\section{EFFECT OF SOLVENT TYPE ON EXTRACTION OF TPC}

The extraction of polyphenol is influenced by several variables, such as the type of solvent, the temperature, the time, the pretreatment operations, etc. Therefore, the effects of some important variables on TPC were investigated for determining the best conditions for the polyphenol extraction from sage.

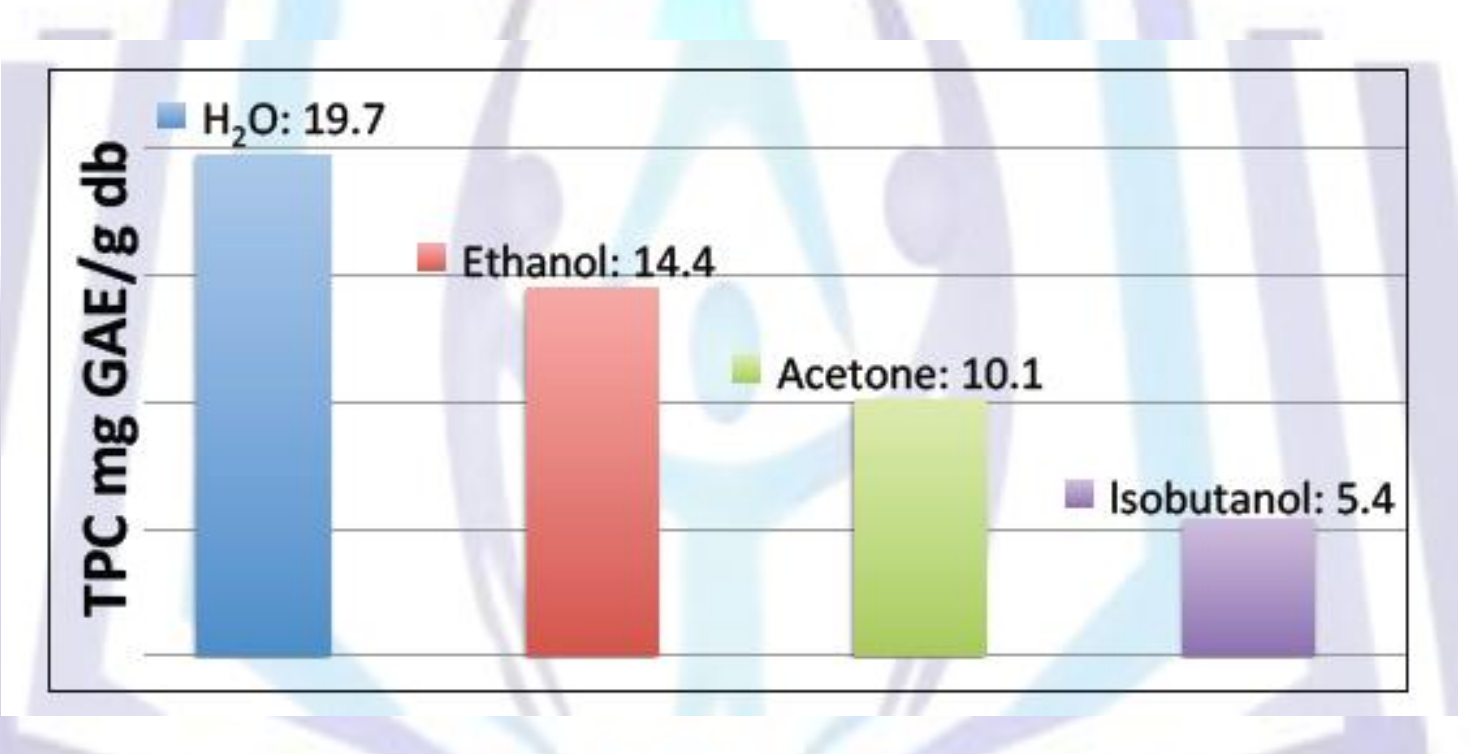

Figure 5. Total phenol values versus solvent type (at 20으, for $20 \mathrm{~min}$ ): Water; Ethanol; Acetone; and Isobutanol.

After 20 min of extraction time, different solvent extraction operations were carried out at ambient temperature (20 $\left.{ }^{\circ} \mathrm{C}\right)$ using water, ethanol, acetone, and isobutanol. The extracted TPC was much higher with water than with the other solvents (Figure 5). Similar results were found in Finland by Dorman, Peltoketo, Hiltunen and Tikkanen [20] and Ollanketo, Peltoketo, Hartonen, Hiltunen and Riekkola [21]. They suggested water to be the most efficient to extract phenolic constituents. Furthermore addition of small quantity of water to organic solvent usually creates a more polar medium which facilitates the extraction of polyphenols [22].

\section{KINETICS OF AQUEOUS EXTRACTION}

This study was carried out in order to acquire the evolution of TPC extraction versus time for raw sage and DIC-treated sage using water as a solvent. As defined by[18], and fromFigure 6,TPC extraction kinetics can be traduced by the starting accessibility $\left(\delta X_{s}=24 \mathrm{mg} / \mathrm{g}\right.$ db for DIC-treated material against $14 \mathrm{mg} / \mathrm{g}$ db for raw material) and by the effective diffusivity $D_{\text {eff. }}$ The TPCyields were much higher when the leaves were DIC treated, to be 35 against $25 \mathrm{mg} / \mathrm{g}$ db for raw untreated material. This increase in TPC yields a shorter time for DIC treated sage is very important in terms of economic and industrial contents. It is clear that DIC-pretreatment is a very relevant intensification way for the total phenol extraction. 


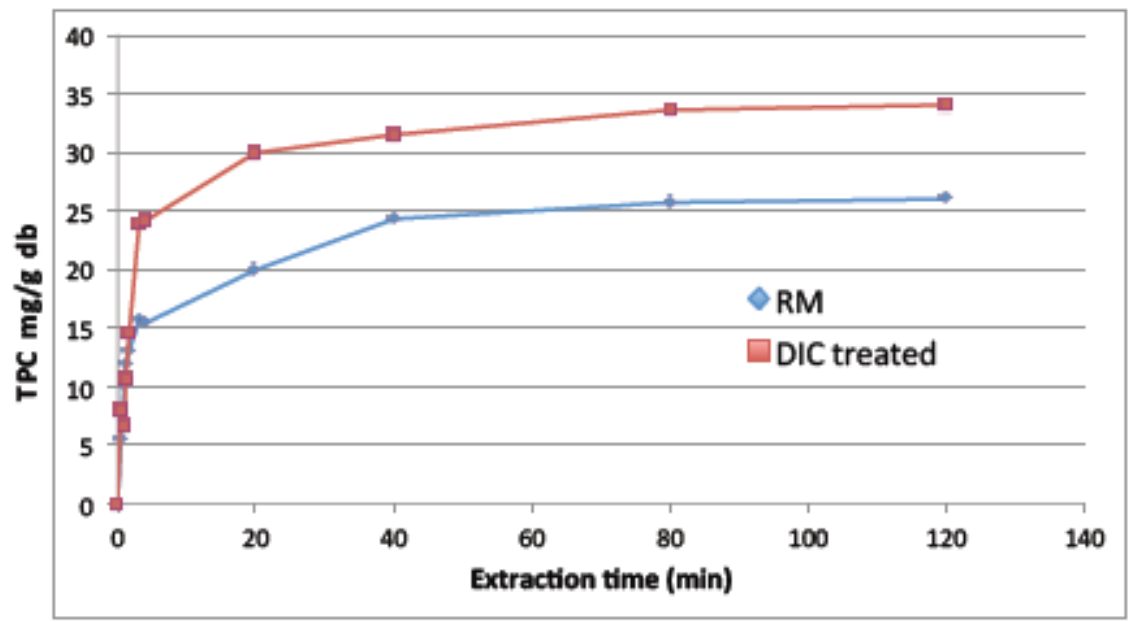

Figure 6.Kinetics profiles for TPC extracted from raw material and DIC-5 treated sample.

\section{STATISTICAL STUDY AND DESIGN OF EXPERIMENT FOR TPC DIC OPTIMIZATION}

Response Surface Methodology RSM can be usually used to optimize various processes in the field of various industrial sectors $[18,23,24]$.

In the present work,RSM was applied as a statistical analysis method to optimize the independent DIC variables (processing pressure and heating time), and to determine the lack of fit and the significance of the linear, quadratic and cross-parameter effects of the independent variables on TPC.

Data of the central composite experimental design and the estimated values of the TPC response were summarized and defined versus the DIC steam pressure and treatment time; they showed relatively higher TPCcompared to those of the raw material. The total amount of phenolic compounds from the extracts of DIC treated samples ranged up to $83 \mathrm{mg}$ GA/ $\mathrm{g}$ $\mathrm{db}$ compared to $23 \mathrm{mg} \mathrm{GA} / \mathrm{g}$ for raw material.

The response surface analysis data for the individual dependent variables for the raw and DIC treated samples are represented in Figure 7. The Pareto charts of standardized effects showed significant effects of pressure (linear and quadratic); the response surface plots can be generated by holding constant one independent variable.

An empirical polynomial quadratic model was calculated for the response TPC from the equations containing six estimated coefficients with the coefficients of determination $R^{2}=90 \%$. The lack of fit test is a measure of the failure of a model to represent data in the experimental domain at which points were not included in the regression, it gives:

$$
\text { TPC }=48.3128-484.689 P+1.96448 t+930.718 P^{2}+3.55257 P t-0.0361517 t^{2}
$$

The high regression factor indicates that the model is adequate and the predictive data fit the experimental results. The processing pressure seemed significant and its linear effect is positive which refers that TPC yields increase with DIC processing pressure. In addition the quadratic effect of DIC heating time is also significant.
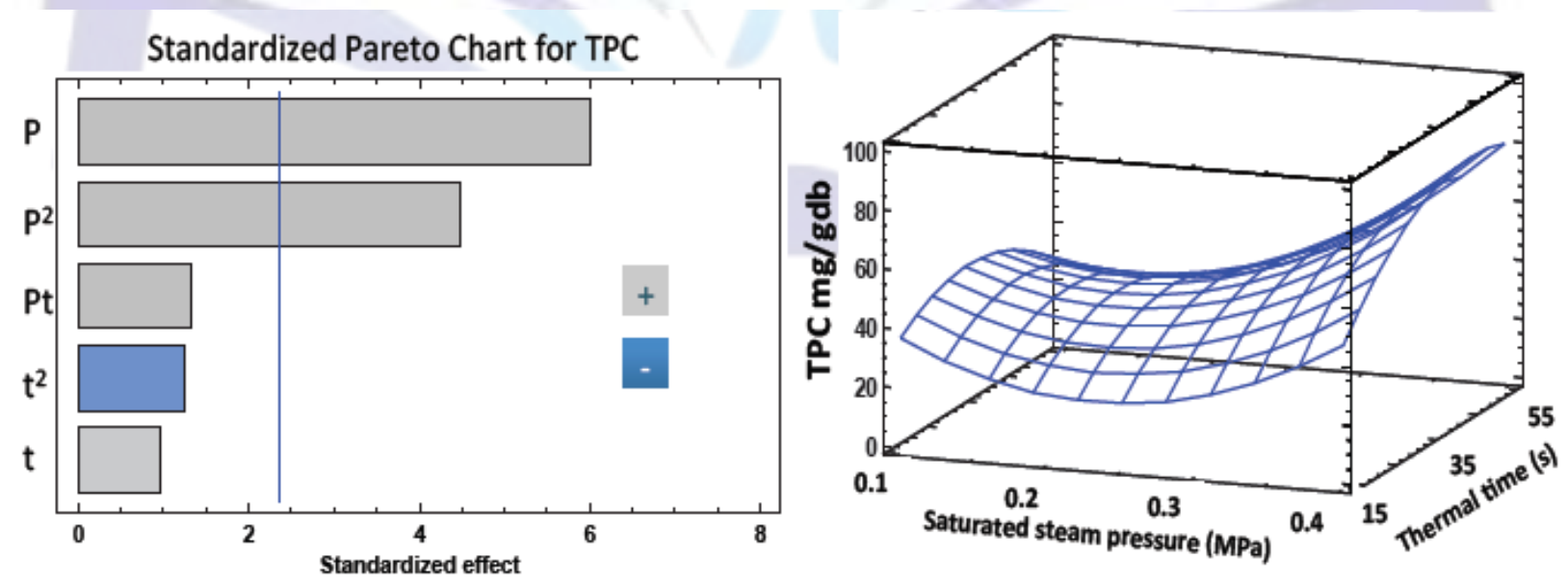

Figure 7.Effects of DIC operating parameters; Pressure (MPa) and time (s) on the TPC: (left) Pareto Chart and (right) response surface 


\section{IMPACT OF DIC TREATMENT ON MICROSTRUCTURE OF SAGE LEAVES}

The SEM images of sage before and after DIC treatment revealed that the microstructure is considerably different.
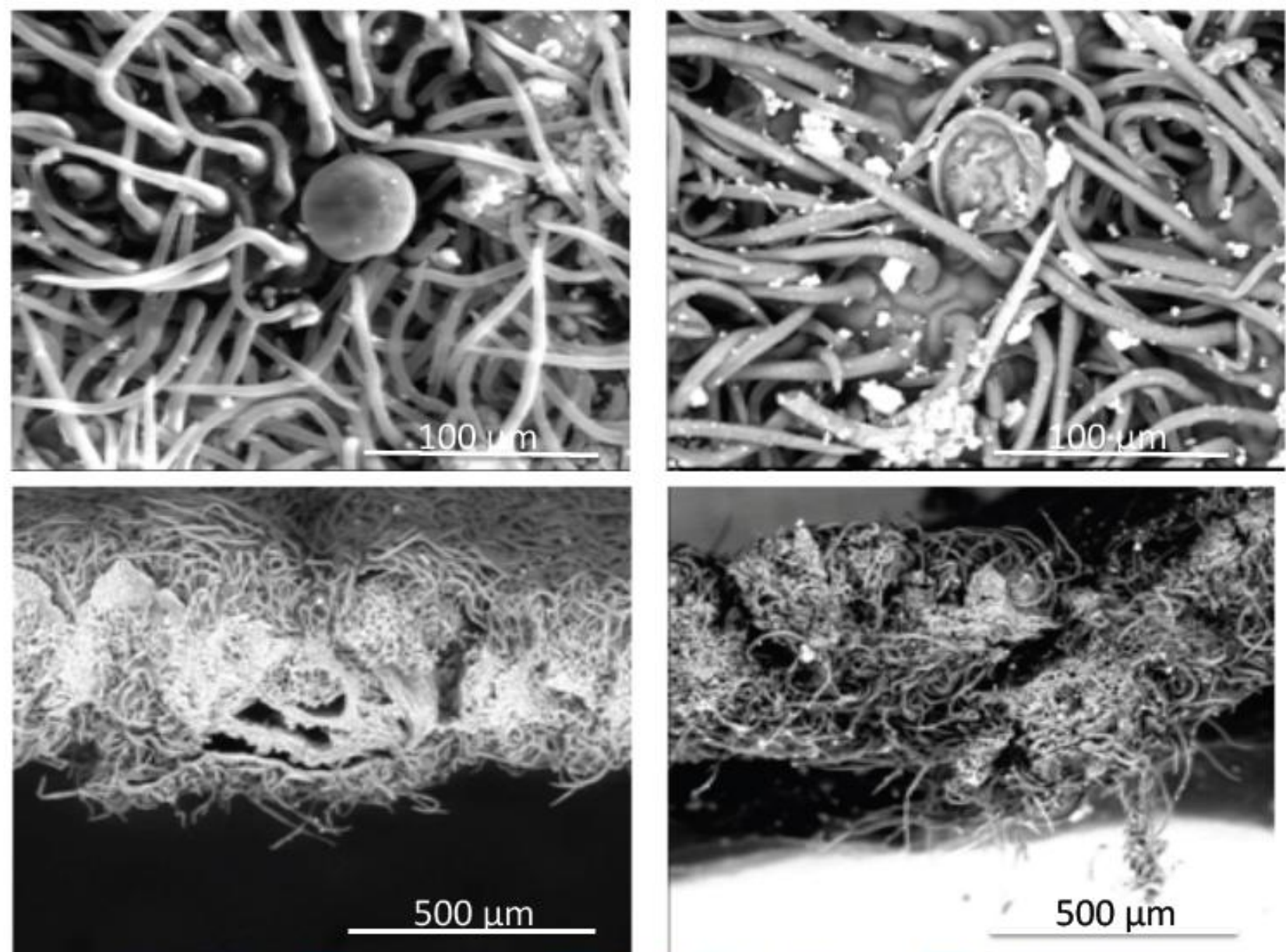

Untreated raw material sage

Figure 8.Scanning Electron Micrographs of Salvia officinalis leaves

The increase in sample volume of microstructure have been due to the treatment of leaves by DIC, the internal vapor pressure creates great mechanical solicitation against the walls, resulting in expansion, which produces a higher porosity microstructure (Figure 8). Similar results were observed by Allaf et al [25] when studying texturing by DIC. DIC treatment can modify the structure of plant at various and controlled levels this will depend on the operating parameters of pretreatment of material [26].

\section{CONCLUSION}

In the present study, sage leaves were tested in order to increase the availability and intensify the extraction capacity of their total phenolic content. The measurements of phenolic compounds in the plant and in the extracts were carried out using the Folin-Ciocalteu method. Conventional extraction method was studied at $20^{\circ} \mathrm{C}$ for 20 min with the objective to compare and find the most adequate between four solvents: water; ethanol; acetone; and isobutanol. Water has presented the best capacity of extraction of polyphenols from sage. Moreover, according to the impact of "Instant controlled pressure drop" DIC, standard solvent extraction applied on textured and expanded plant indicated much higher availability and yields, as well as better rate and lower extraction time. Decisively, the results of the present study indicate that the tested sage can be a real source of plant-based natural polyphenols. By establishing and optimizing the DIC-assisted water extraction sage can be efficient sources of polyphenols. Finally, it was clear that the structure modification of DICexpanded textured sage allows the hot air drying to trigger greater kinetics.

\section{REFERENCES}

[1] B. Tomaz, B. Dea, The Biological/Pharmacological Activity of the Salvia Genus, in: Sage, CRC Press, 2000, pp. 143184. 
[2] B. Tepe, O. Eminagaoglu, H.A. Akpulat, E. Aydin, Antioxidant potentials and rosmarinic acid levels of the methanolic extracts of Salvia verticillata (L.) subsp. verticillata and S. verticillata (L.) subsp. amasiaca (Freyn \&amp; Bornm.) Bornm, Food Chemistry, 100 (2007) 985-989.

[3] S.L. Cuppett, C.A. Hall, 3rd, Antioxidant activity of the Labiatae, Advances in food and nutrition research, 42 (1998) 245-271.

[4] K. Miura, H. Kikuzaki, N. Nakatani, Antioxidant activity of chemical components from sage (Salvia officinalis L.) and thyme (Thymus vulgaris L.) measured by the oil stability index method, J Agric Food Chem, 50 (2002) 1845-1851.

[5] A. Zainuddin, J. Pokorny, R. Venskutonis, Antioxidant activity of sweetgrass (Hierochloe odorata Wahlnb.) extract in lard and rapeseed oil emulsions, Die Nahrung, 46 (2002) 15-17.

[6] M. Ozcan, Antioxidant activities of rosemary, sage, and sumac extracts and their combinations on stability of natural peanut oil, Journal of medicinal food, 6 (2003) 267-270.

[7] I. Jaswir, Y.B. Che Man, T.H. Hassan, Performance of phytochemical antioxidant systems in refined-bleacheddeodorized palm olein during frying, Asia Pacific journal of clinical nutrition, 14 (2005) 402-413.

[8] L.-N. Li, Biologically active components from traditional Chinese medicines Pure and Applied Chemistry, 70 (1998) 547-554.

[9] M. Andrew, H. Kurt, Separation and Quantification of Flavonoids, in: Flavonoids, CRC Press, 2005, pp. 1-36.

[10] M. Koşar, H.J.D. Dorman, R. Hiltunen, Effect of an acid treatment on the phytochemical and antioxidant characteristics of extracts from selected Lamiaceae species, Food Chemistry, 91 (2005) 525-533.

[11] N.E. Durling, O.J. Catchpole, J.B. Grey, R.F. Webby, K.A. Mitchell, L.Y. Foo, N.B. Perry, Extraction of phenolics and essential oil from dried sage (Salvia officinalis) using ethanol-water mixtures, Food Chemistry, 101 (2007) 1417-1424.

[12] K. Allaf, Approche à l'analyse fondamentale de l'expansion par alvéolation selon différents procédés (puffing, cuissonextrusion...), in, Université de Technologie de Compiègne, Compiègne - France, 1988.

[13] B. Ben Amor, K. Allaf, Impact of texturing using instant pressure drop treatment prior to solvent extraction of anthocyanins from Malaysian Roselle (Hibiscus sabdariffa), Food Chemistry, 115 (2009) 820-825.

[14] M. Kristiawan, V. Sobolik, M. Al-Haddad, K. Allaf, Effect of pressure-drop rate on the isolation of cananga oil using instantaneous controlled pressure-drop process, Chemical Engineering and Processing: Process Intensification, 47 (2008) 66-75.

[15] B. Berka-Zougali, A. Hassani, C. Besombes, K. Allaf, Extraction of essential oils from Algerian myrtle leaves using instant controlled pressure drop technology, Journal of Chromatography A, 1217 (2010) 6134-6142.

[16] C. Besombes, B. Berka-Zougali, K. Allaf, Instant controlled pressure drop extraction of lavandin essential oils: fundamentals and experimental studies, Journal of Chromatography A, 1217 (2010) 6807-6815.

[17] S. Mounir, C. Besombes, N. Al-Bitar, K. Allaf, Study of Instant Controlled Pressure Drop DIC - Treatment in Manufacturing Snack and Expanded Granule Powder of Apple and Onion, Drying Technology, 29 (2011) 331-331.

[18] T. Allaf, K. Allaf, Instant Controlled Pressure Drop (D.I.C.) in Food Processing, Springer, New York, 2014.

[19] V.L. Singletong, J.A. Rossi, Colorimetry of total phenolics with phosphomolybdic-phosphotungstic acid reagents, American Journal of Enology Viticulture, 16 (1965) 144-158.

[20] H.J.D. Dorman, A. Peltoketo, R. Hiltunen, M.J. Tikkanen, Characterisation of the antioxidant properties of deodourised aqueous extracts from selected Lamiaceae herbs, Food Chemistry, 83 (2003) 255-262.

[21] M. Ollanketo, A. Peltoketo, K. Hartonen, R. Hiltunen, M.-L. Riekkola, Extraction of sage (Salvia officinalis L.) by pressurized hot water and conventional methods: antioxidant activity of the extracts, Eur Food Res Technol, 215 (2002) 158-163.

[22] G. Spigno, L. Tramelli, D.M. De Faveri, Effects of extraction time, temperature and solvent on concentration and antioxidant activity of grape marc phenolics, Journal of Food Engineering, 81 (2007) 200-208.

[23] S. Tsapatsaris, P. Kotzekidou, Application of central composite design and response surface methodology to the fermentation of olive juice by Lactobacillus plantarum and Debaryomyces hansenii, Int J Food Microbiol, 95 (2004) 157-168.

[24] M. Noshad, M. Mohebbi, F. Shahidi, S. Ali Mortazavi, Multi-Objective Optimization of Osmotic-Ultrasonic Pretreatments and Hot-Air Drying of Quince Using Response Surface Methodology, Food and Bioprocess Technology, 5 (2012) 2098-2110.

[25] T. Allaf, V. Tomao, K. Ruiz, K. Bachari, M. EIMaataoui, F. Chemat, Deodorization by instant controlled pressure drop autovaporization of rosemary leaves prior to solvent extraction of antioxidants, LWT - Food Science and Technology, 51 (2013) 111-119. 
[26] B.B. Amor, C. Lamy, P. Andre, K. Allaf, Effect of instant controlled pressure drop treatments on the oligosaccharides extractability and microstructure of Tephrosia purpurea seeds, Journal of Chromatography A, 1213 (2008) 118-124.

Author' biography with Photo

\section{RESEARCH \& EDUCATION THEMES}

Born on February 15, 1952, in Tripoli (Lebanon).

Nationality:

Present: French.

Birth: Lebanese.

Prof. Karim ALLAF, (Professor Exceptional Class), Mentor of research team of "Intensification of Transfer Phenomena on Industrial Eco-Processes", at Laboratory

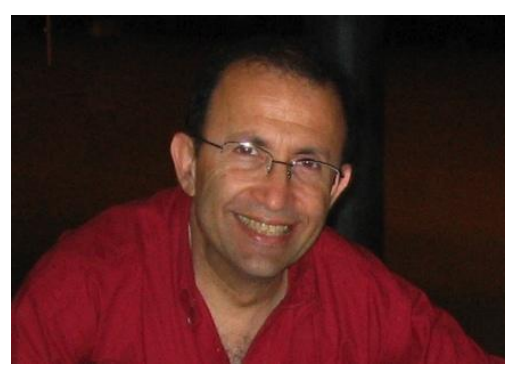
of Engineering Science for Environment FRE 3474 CNRS (France), born on February 15th, 1952, in Tripoli in Lebanon, was graduated in 1976 from the University of Paris-XI University where he primed a Doctorate in Physics of low temperature Plasmas, after which he spent five years as a Researcher at the French CNRS completing a PhD in Thermodynamics and Chemical Engineering in 1981 before commencing a career as an Associated Professor, Director of the Department of Physics of the Faculty of Science in Lebanese University (1981/1988), and as Associated Professor then Professor, scientific responsible of Agro-industrial Technologies research team DTAl at the Department of Chemical Engineering of the University of Technology of Compiegne UTC (1988/1994). In 1994, Prof. Allaf joined La Rochelle University to create and head the Department of Process Engineering, the Institute of Industrial Process and Equipment, and the Laboratory of Mastering Technologies for Agro-Industry LMTAI where he has developed specific activities on the technologies of Instantaneous Thermodynamics. He and collaborators have defined, optimized, and transferred towards various industrial sectors the technology of instant controlled pressure drop DIC, using it in drying, texturing, extraction and decontamination operations. His research team completed work by defining innovative processes of Drying (swelldrying, three-stage expanded granule spray-drying...), and outlining innovative intermittent ways for drying (Multi Flash Drying MFD and Intermittent Air-Flow IAF drying). They have defined novel unit operations of extraction, designing adequate equipment for industrial scale applications. Recently, Pr. ALLAF and collaborators in La Rochelle started studies on DIC-assisted in situ transesterification of canola, microalgae... as well as new intensifications of acid and enzymatic hydrolysis of cellulose compounds. Prof. ALLAF has got 12 Patents and 24 extensions (concerning mainly the instant controlled pressure drop DIC technology), 115 international papers, 5 books, 158 full-communications in international congresses, and numerous European and industrial reports (34). Pr. ALLAF has been the mentor of $31 \mathrm{PhD}$ workst; he has been the main mentor of $31 \mathrm{PhD}$ and $6 \mathrm{HDR}$ researcher works, and the coordinator of 8 European projects since 1993. Mr. Allaf participated to various activities on transferring technologies towards the industry. He created the Company ABCAR-DIC Process, in France, and greatly contributed to the creation of various international enterprises such as Nutrimezza, in Queretaro (Mexico), Bio-essential and ABCAR-Malaysia, in Malaysia, Bio-Golden, in Spain, etc. He also contributed to the creation of ULR-Valor to intensify the collaboration between the University of La Rochelle and various industry sectors..

La Rochelle, le 18/01/2014

Professor Karim ALLAF

Former Director of Laboratory Mastering Technologies for Agro-Industry LMTAI

Former Director of department of Process Engineering

Former Director of IUP Industrial Engineering of Equipment and Processes GEPI 